\title{
Indirect pathological indicators for cardiac sarcoidosis on endomyocardial biopsy
}

\author{
Myung-Jin Cha ${ }^{1}$, Jeong-Wook $\mathrm{Seo}^{2}$, Seil Oh ${ }^{3,4}$, Eun-Ah Park ${ }^{5}$, Sang-Han Lee ${ }^{6,7}$, Moon Young Kimº ${ }^{8}$, Jae-Young Park ${ }^{9}$ \\ 'Division of Cardiology, Department of Internal Medicine, Seoul National University Hospital, Seoul; \\ ${ }^{2}$ Department of Pathology and ${ }^{3}$ Department of Internal Medicine and Interdisciplinary Program for Bioengineering, Seoul National University College of Medicine, Seoul; \\ ${ }^{4}$ Cardiology Division, Cardiovascular Center, and Cardiac Electrophysiology Lab, Seoul National University Hospital, Seoul; \\ ${ }^{5}$ Division of Cardiovascular Imaging, Department of Radiology, Seoul National University Hospital, Seoul; \\ ${ }^{6}$ Department of Forensic Medicine, School of Medicine, Kyungpook National University, Daegu; \\ ${ }^{7}$ Department of Pathology, Kyungpook National University Hospital, Daegu; \\ ${ }^{8}$ Department of Radiology, Seoul Metropolitan Government - Seoul National University Boramae Medical Center, Seoul; \\ 9Department of Pathology, Sejong Hospital, Bucheon, Korea
}

Background: The definitive pathologic diagnosis of cardiac sarcoidosis requires observation of a granuloma in the myocardial tissue. It is common, however, to receive a "negative" report for a clinically probable case. We would like to advise pathologists and clinicians on how to interpret "negative" biopsies. Methods: Our study samples were 27 endomyocardial biopsies from 25 patients, three cardiac transplantation and an autopsied heart with suspected cardiac sarcoidosis. Pathologic, radiologic, and clinical features were compared. Results: The presence of micro-granulomas or increased histiocytic infiltration was always (6/6 or $100 \%)$ associated with fatty infiltration and confluent fibrosis, and they showed radiological features of sarcoidosis. Three of five cases $(60 \%)$ with fatty change and confluent fibrosis were probable for cardiac sarcoidosis on radiology. When either confluent fibrosis or fatty change was present, one-third (3/9) were radiologically probable for cardiac sarcoidosis. We interpreted cases with micro-granuloma as positive for cardiac sarcoidosis (five of $25,20 \%$ ). Cases with both confluent fibrosis and fatty change were interpreted as probable for cardiac sarcoidosis (seven of 25 , 28\%). Another 13 cases, including eight cases with either confluent fibrosis or fatty change, were interpreted as low probability based on endomyocardial biopsy. Conclusions: The presence of micro-granuloma could be an evidence for positive diagnosis of cardiac sarcoidosis. Presence of both confluent fibrosis and fatty change is necessary for probable cardiac sarcoidosis in the absence of granuloma. Either of confluent fibrosis or fatty change may be an indirect pathological evidence but they are interpreted as nonspecific findings.

Key Words: Myocarditis; Arrhythmogenic right ventricular dysplasia; Tachycardia, ventricular; Sarcoidosis; Cardiac muscle

Received: December 17, 2019 Revised: June 2, 2020 Accepted: June 10, 2020

Corresponding Author: Jeong-Wook Seo, MD, Department of Pathology, Seoul National University College of Medicine, 103 Daehak-ro, Jongno-gu, Seoul 03080, Korea Tel: +82-2-740-8268, Fax: +82-2-765-5600, E-mail: jwseo@snu.ac.kr

Cardiac sarcoidosis is a myocardial inflammatory disease with non-caseating granulomas [1]. Cardiac sarcoidosis may be cardiac involvement of systemic sarcoidosis, although the heart may be the only organ involved. In contrast to pulmonary sarcoidosis, cardiac involvement of sarcoidosis is often associated with fatal outcome because of ventricular arrhythmia and ventricular dysfunction $[2,3]$. Clinical studies reveal cardiac involvement in $5 \%-10 \%$ of systemic sarcoidosis cases [4] but others suggest this involvement to be very rare $(0.7 \%)[5]$ or very common ( $40 \%)$ based on symptomology [6]. The incidence in autopsy series was $20 \%$ in a 1952 study [7], but recent studies have demonstrated variable incidences of $27 \%$ [8], $76 \%$ in Caucasian patients [4], and $80 \%$ in Japanese patients [9]. This variability in incidence may be real; partly due to racial differences in prevalence and due to the development of diagnostic techniques such as the endobronchial ultrasound or cardiac positron emission tomography (PET) [10]. But it may also be related to the variable application of the current diagnostic criteria $[1,11]$.

With respect to the pathologic diagnosis of cardiac involvement in systemic sarcoidosis, "the presence of non-caseating granuloma on histological examination of myocardial tissue with no alternative cause identified" is the current consensus by the Heart 
Rhythm Society (HRS) [12]. Although the statement by the HRS is the most reliable description on cardiac involvement in systemic sarcoidosis $[2,3,13]$, its use for pathological diagnosis is limited. Positive diagnosis on endomyocardial biopsy is the only way to confirm cardiac involvement in the current system. The clinical diagnosis of "probable involvement" requires histologic confirmation of extracardiac sarcoidosis. The Japanese Guidelines for Diagnosis of Cardiac Sarcoidosis based on the Study Report on Diffuse Pulmonary Diseases by the Japanese Ministry of Health is more practical for clinical decisions, and the presence of "interstitial fibrosis or monocyte infiltrate over moderate grade" was included as additional minor criteria $[10,14]$. Based on the current statement, we can only confirm the diagnosis of cardiac sarcoidosis when a granuloma is observed.

Our current pathologic diagnostic practice is somewhat different from the clinical guidelines $[12,14]$ and reported autopsy experiences $[4,7-9]$ because we seldom find definitive granulomas in endomyocardial biopsies of those with clinical cardiac sarcoidosis. Several measures are undertaken to increase the possibility of detecting granuloma; robot-guided biopsy [15], electrocardiogram guided biopsy [16], electro-anatomical mapping system and intracardiac echography guided biopsies [17] are examples. What would be the role of endomyocardial biopsy when we can't find granulomas despite these efforts, but the clinical findings are compatible with cardiac sarcoidosis? There are reports of clinical cardiac sarcoidosis associated with biopsy-confirmed systemic sarcoidosis, but without granuloma in the autopsied heart [18]. Therefore, there is a dilemma for pathologic diagnosis of sarcoidosis in resected whole hearts when no classical granulomas are observed.

We hypothesized that the current pathologic criteria for cardiac sarcoidosis by endomyocardial biopsies are not sensitive enough to detect clinical cases or late phase of possible cardiac sarcoidosis. We therefore claim that pathologic findings other than granuloma may have some significance for clinical decisions and the management of sarcoidosis patients. We therefore categorized findings on endomyocardial biopsies according to the combination of the presence of four pathologic indicators: micro-granulomas, histiocytic infiltration, confluent fibrosis, and fatty change.

We studied 27 endomyocardial biopsies from 25 patients with suspected clinical cardiac sarcoidosis. We also studied four whole hearts: three resected for cardiac transplantation and one examined by autopsy. Clinical, radiological, and therapeutic responses were compared among these pathologic interpretation groups. Our hope is that this categorization approach will correlate with clinical findings, and that these pathologic findings can provide physicians with more information than the presence or absence of granuloma.

\section{MATERIALS AND METHODS}

We reviewed 27 endomyocardial biopsies and three whole hearts after cardiac transplantation from 25 patients at Seoul National University Hospital from 2012 to May 2017. Cases were collected from those with a clinical diagnosis of cardiac sarcoidosis when endomyocardial biopsy was performed. Clinical suspicion of cardiac sarcoidosis was made when there is an arrhythmia and extracardiac sarcoidosis; or certain types of arrhythmia (the second degree Mobitz type II or the third-degree atrioventricular block with or without radiologic features suggestive of cardiac sarcoidosis). We included consecutive cases with different levels of probability suspected by the physician. Clinical summary of 27 biopsies from 25 patients are described in Table 1 .

\section{Interpretation criteria of magnetic resonance imaging}

The magnetic resonance imaging (MRI) findings were categorized as follows: (1) probable cases: these cases involve multiple patchy enhancement at the subepicardial or mid-myocardial layers, which do not correspond to the expected territories of coronary arterial supply. Involvement of the basal part of the interventricular septum, irregular thinning of the wall, and ejection fraction less than $50 \%$ are considered probable cases; (2) possible cases: these cases have features similar to those of probable cases, but diseases other than cardiac sarcoidosis could be considered a cause of the lesion; (3) nonspecific findings: these cases have minor and focal lesions of fibrosis, which may be interpreted as early lesions of sarcoidosis or other conditions; and (4) unlikely: these cases have radiological features more likely to indicate an ischemic condition or other specific conditions.

\section{Interpretation criteria of four pathologic findings}

The endomyocardial biopsies were stained with hematoxylin and eosin stain, Masson's trichrome (MT) stain, and immunohistochemical stain for CD68. The pathological findings were analyzed based on four morphological changes: micro-granulomas, increased histiocytes, confluent fibrosis, and fatty tissue. Subsequently, cases were described and analyzed according to groups of morphologic findings.

\section{Micro-granuloma}

A granuloma is defined as a collection of histiocytes. The size of granulomas vary and there is no consensus on the minimum size of granuloma, although it is generally accepted that the gran- 
Table 1. Summary of information on cases of clinically suspected cardiac sarcoidosis

\begin{tabular}{|c|c|c|c|c|c|c|c|c|c|c|}
\hline Case No. ${ }^{a}$ & Sex & $\mathrm{Age}^{\mathrm{b}}$ & Extracardiac sarcoidosis & $\begin{array}{l}\text { Steroid } \\
\text { effect }\end{array}$ & Cardiac rhythm & Heart block ${ }^{\circ}$ & $\begin{array}{l}\text { Ventricular } \\
\text { arrhythmia }^{d}\end{array}$ & LVEF (\%) & Heart TPL & $\begin{array}{c}\text { MRI } \\
\text { diagnosis }\end{array}$ \\
\hline \multicolumn{11}{|l|}{ Group 1} \\
\hline $1-1$ & M & $>40$ & No & No & Sinus & No & No & 22 & Yes & Probable \\
\hline $1-2$ & M & $>40$ & No & No & Sinus & No & No & 33 & Yes & Probable \\
\hline $1-3$ & $\mathrm{~F}$ & $>50$ & Not definite & No & Sinus & No & No & 51 & No & Probable \\
\hline $1-4$ & M & $>60$ & No & Unknown & Paroxysmal AF & No & No & 30 & No & $x$ \\
\hline $1-5$ & $\mathrm{~F}$ & $>60$ & No & No & Sinus & No & No & 42 & No & Probable \\
\hline \multicolumn{11}{|l|}{ Group 2} \\
\hline $2-1$ & $\mathrm{~F}$ & $>70$ & No & No & $\mathrm{AF}$ & No & No & 15 & No & Probable \\
\hline $2-2$ & $\mathrm{~F}$ & $>70$ & No & No & AF & No & No & 15 & No & Probable \\
\hline \multicolumn{11}{|l|}{ Group 3} \\
\hline $3-1$ & M & $>50$ & No & No & Sinus & Yes & Yes & 44 & No & Probable \\
\hline $3-2$ & $\mathrm{~F}$ & $>70$ & No & Yes & Paroxysmal AF & Yes & No & 40 & No & Possible \\
\hline $3-3$ & M & $>40$ & No & No & Sinus & Yes & Yes & 36 & Yes & Nonspecific \\
\hline $3-4$ & $\mathrm{~F}$ & $>90$ & No & Unknown & Sinus & Yes & Yes & 30 & No & Probable \\
\hline $3-5$ & $\mathrm{~F}$ & $>50$ & Yes (lung, lymph node) & Unknown & Sinus & Yes & Yes & 50 & No & $x$ \\
\hline $3-6$ & $\mathrm{~F}$ & $>70$ & No & Yes & Sinus & No & No & 22 & No & Probable \\
\hline \multicolumn{11}{|l|}{ Group 4} \\
\hline $4-1$ & M & $>60$ & No & Unknown & Sinus & No & Yes & 30 & Yes & Probable \\
\hline $4-2$ & M & $>40$ & No & No & Sinus & No & No & 33 & Yes & Probable \\
\hline $4-3$ & M & $>70$ & No & Unknown & $\mathrm{AF}$ & No & No & 56 & No & Nonspecific \\
\hline \multicolumn{11}{|l|}{ Group 5} \\
\hline $5-1$ & M & $>70$ & No & No & Sinus & No & No & 48 & No & Probable \\
\hline $5-2$ & $\mathrm{~F}$ & $>60$ & No & Yes & Sinus & No & No & 35 & No & Nonspecific \\
\hline $5-3$ & $F$ & $>70$ & No & Yes & Paroxysmal AF & No & Yes & 35 & No & Nonspecific \\
\hline $5-4$ & M & $>60$ & No & No & Sinus & No & No & 48 & No & Nonspecific \\
\hline $5-5$ & M & $>70$ & Yes (lymph node) & No & Sinus & No & Yes & 42 & No & Unlikely \\
\hline $5-6$ & $\mathrm{~F}$ & $>50$ & Yes (skin) & Yes & Sinus & Yes & No & 61 & No & Unlikely \\
\hline \multicolumn{11}{|l|}{ Group 6} \\
\hline $6-1$ & M & $>50$ & No & Unknown & Sinus & Yes & Yes & 46 & No & $x$ \\
\hline $6-2$ & M & $>30$ & No & No & Sinus & No & No & 30 & No & Nonspecific \\
\hline $6-3$ & M & $>20$ & Yes (lymph node) & Yes & Sinus & No & No & 25 & No & Nonspecific \\
\hline $6-4$ & M & $>60$ & No & Unknown & Sinus & No & No & 28 & No & Nonspecific \\
\hline $6-5$ & $\mathrm{M}$ & $>10$ & No & Unknown & Sinus & No & No & 54 & No & Unlikely \\
\hline
\end{tabular}

Group 1, endomyocardial biopsies with micro-granuloma as well as histiocytic infiltration, confluent fibrosis and fatty change; Group 2, endomyocardial biopsies with histiocytic infiltration, confluent fibrosis and fatty change but without micro-granuloma; Group 3, endomyocardial biopsies with confluent fibrosis associated with fatty tissue infiltration; Group 4, presence of confluent fibrosis without associated fatty tissue; Group 5, presence of fatty tissue without associated fibrosis; Group 6, none of four possible indicators on endomyocardial biopsy.

LVEF, left ventricular ejection fraction; TPL, transplantation; MRI, magnetic resonance imaging: M, male; F, female; AF, atrail fibrillation.

${ }^{a}$ Cases 1-2 and 4-2 are a same case, cases 2-1 and 2-2 are another same case; 'Age is expressed in 10-year interval; " cSecond degree Mobitz type II or thirddegree atrioventricular block; 'Sustained ventricular tachycardia or ventricular fibrillation.

uloma has a size more than 30 or 50 histiocytes. In this study, we interpreted a small nodular collection of 5-10 histiocytes as a micro-granuloma. CD68 staining was necessary to find a microgranuloma (Fig. 1).

\section{Increased histiocytes}

In some cases, infiltration of histiocytes was observed at the myocardial interstitium. We classified a significant increase in histiocytes in the interstitium when histiocytes are at least three times more than their usual frequency. CD68 immunostaining was necessary to count the number of histiocytes.

\section{Confluent fibrosis}

Confluent fibrosis replacing more than 30-50 myocardial cells was considered as a significant post-necrotic lesion in this study. The fibrotic lesion was more or less edematous and was associated with some inflammatory cells. We did not count the slender collagen fibers in the interstitium without myocyte damage, which was commonly encountered as interstitial fibrosis. Multifocal spotty fibrosis following necrosis of individual myocardial cells 


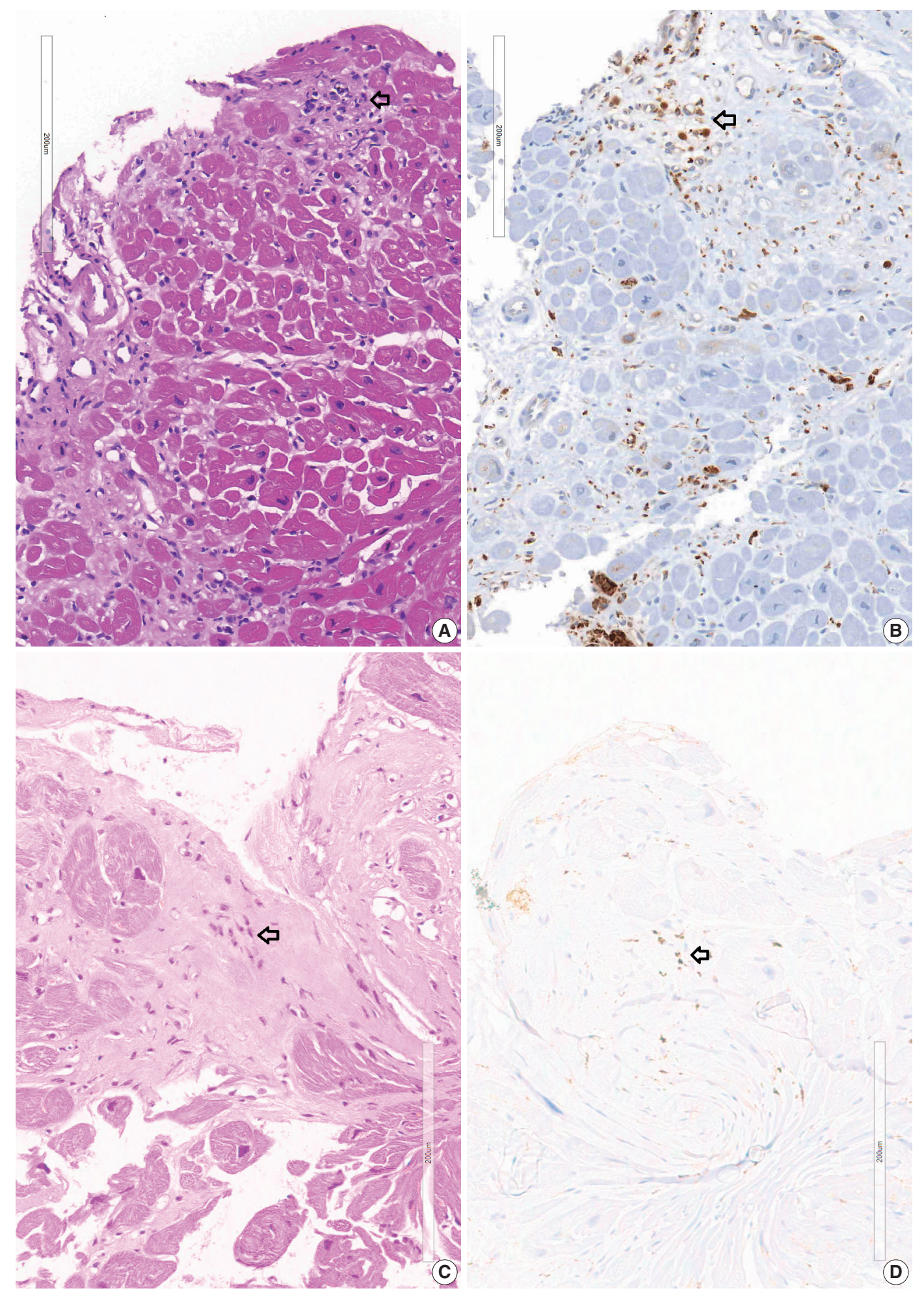

Fig. 1. Micro-granuloma on the endomyocardial biopsy. (A) Endomyocardial biopsy at 2 years prior to the transplantation of case 1-1 shows confluent fibrosis with edematous stroma. Three foci of infiltration of histiocytes and lymphocytes (arrow) are seen at the margin of fibrosis which is the interface between the fibrosis and myocardium. (B) CD68 staining of the same specimen showing histiocytic infiltration at the micro-granulomas (arrow). (C) Endomyocardial biopsy of case 1-3 shows a micro-granuloma (arrow) of 15 cells in the fibrotic zone. (D) CD68 immunostaining of endomyocardial biopsy of case 1-3 shows positive staining (arrow) on histiocytic marker. 
(replacement fibrosis) was also excluded in this study. MT stain was useful to reveal confluent fibrosis.

\section{Fatty change}

Post-inflammatory infiltrated fat cells were also recognized as individual fat cells in the fibrotic myocardial scar. Subendocardial accumulation of fat cells was also observed. Sometimes young fat cells with small and bubbly cytoplasm were noted. Differentiating them from fatty tissue as a normal component of the interstitium between myocardial bundles and perivascular space or as an extension of epicardial fatty tissue was difficult. Therefore, we noted infiltrations of fatty tissue of more than 10 adipocytes, which were interpreted as the presence of fatty tissue in this study.

Four cases received cardiac transplantation and three transplant hearts were examined by conventional gross dissection of the heart. A semi-quantitative histological mapping was performed on a sectional plane of a heart. Pathologic features were examined to determine granulomas and other related pathologic findings. An autopsied heart was examined, and microscopic images were added as supplementary material to compare classical sarcoidosis before treatment with our clinical cases in endomyocardial biopsies and heart transplantation.

\section{RESULTS}

\section{Histopathologic findings of 27 endomyocardial biopsies}

\section{Endomyocardial biopsies with micro-granuloma as well as histiocytic infiltration, confluent fibrosis, and fatty change}

Five endomyocardial biopsies were included in group 1 (Table 1). None of the five endomyocardial biopsies were interpreted as granuloma in the initial pathologic report because of small atypical granuloma-like collections of 5-10 histiocytes. We interpreted these cases as micro-granulomas based on the current definition for this study. One case was reported as myocarditis and four others had confluent fibrosis associated with increased histiocytes. One case was the second endomyocardial biopsy after the first biopsy showed no micro-granulomas two years previously. No cases had any evidence of extracardiac sarcoidosis. All five cases showed confluent fibrosis, increased histiocytes, and fatty change (Fig. 1). Two cases received cardiac transplantation after which the resected hearts showed granulomas (Fig. 2).

Four of these patients had MRI findings indicating probable sarcoidosis. One case was not checked for cardiac MRI but clinical findings were suggestive of cardiac sarcoidosis; global hypo-
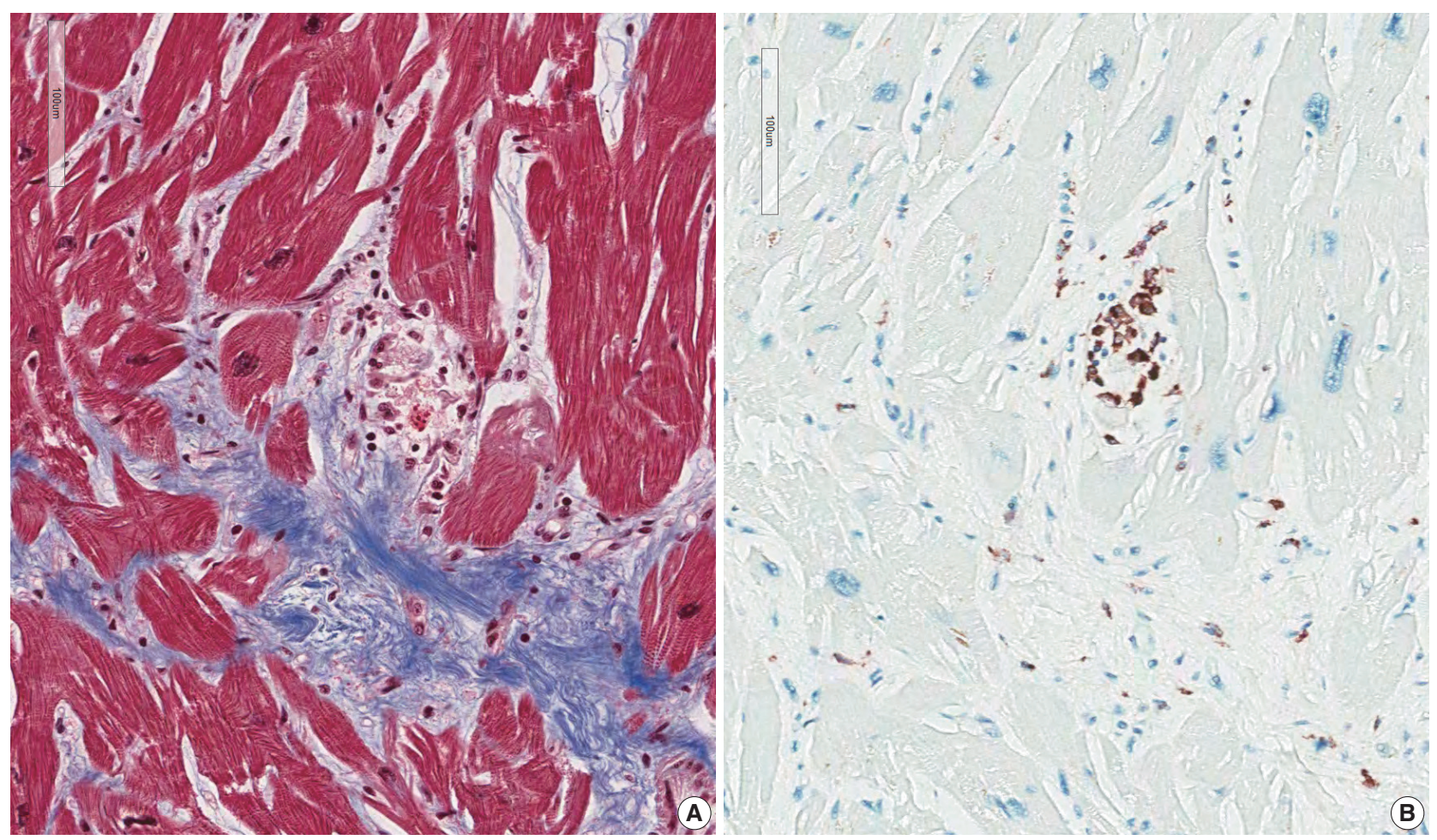

Fig. 2. Micro-granuloma in an explant heart. (A) Magnification of the ventricular myocardium of explant heart in case 1-2 reveals interstitial fibrosis and a few small granulomas. (B) Immunohistochemical staining on CD68 in the same area shows a small nodular collection of histiocytes or zone of scattered histiocytes. 
kinesia on the echocardiography and low left ventricular ejection fraction $(30 \%)$ without any evidence of ischemic heart disease.

\section{Endomyocardial biopsies with histiocytic infiltration, confluent fibrosis, and fatty change but without micro-granuloma}

Two endomyocardial biopsies from a patient (group 2) showed an increase in histiocytes, confluent fibrosis, and fatty change. Two endomyocardial biopsies showed confluent fibrosis admixed with fatty change and increased histiocytes in the same area within the biopsied tissue (Fig. 3). Granuloma was not definitive but nodules of 3-5 histiocytes were scattered. MRI findings were compatible with sarcoidosis, although extracardiac evidence of sarcoidosis was lacking.

\section{Endomyocardial biopsies with confluent fibrosis associated with} fatty tissue infiltration

Confluent fibrosis was associated with fatty infiltration in six cases (group 3). Fatty change was observed at the immediate subendocardium or in the fibrous scar. Adipocytes showed variable sizes and were admixed with fibrosis, particularly at the margin of the fatty tissue and at the junction of the myocardium (Fig. 4).

One case was associated with extracardiac sarcoidosis in the lungs and mediastinal lymph nodes. MRI findings of four cases were compatible with sarcoidosis, though one case was excluded. MRI was not performed in two cases. The echocardiographic results of two cases showed unexplained ventricular dysfunction (left ventricular ejection fraction of 33\%) with global hypokinesia and basal septal akinesia with thinning, respectively, which suggested cardiac sarcoidosis.

\section{Presence of confluent fibrosis without associated fatty tissue}

Three cases in group 4 showed confluent fibrosis (Fig. 5). Two cases showed hyalinized fibrosis, and one case showed fibrosis and edematous stroma. Confluent fibrosis is not evident in a small biopsy, but fibrosis larger than five times the diameter of myocardial cells can be interpreted as a scar related to a previous site of granuloma. Perivascular interstitium sometimes expanded to mimic confluent fibrosis and a fibrotic lesion around the blood vessels should be excluded before interpreting post-granuloma scar. Confluent fibrosis may be young edematous lesion, but may also be a hyalinized scar.

\section{Presence of fatty tissue without associated fibrosis}

Six cases in group 5 showed fatty tissue without associated fibrosis. Fatty tissue was an expansion of perivascular fatty tissue or fatty tissue between myocardial muscle groups (Fig. 4D).

One case showed MRI findings consistent with sarcoidosis, whereas three cases showed MRI findings not consistent with sarcoidosis. Two other cases had sarcoidosis in the mediastinal lymph node or skin, but the MRI findings did not support sarcoidosis.

\section{None of four possible indicators on endomyocardial biopsy}

Six cases showed normal myocardium or only minimal interstitial fibrosis (group 6). Three cases showed nonspecific findings on MRI. Two cases showed unlikely diagnosis on MRI. One case did not have MRI results, but the echocardiography showed normal left ventricular function with unexplained basal septal akinesia with thinning, because of which cardiac sarcoidosis was suspected.

\section{Pathology of three cases of cardiac transplantation and an autopsy case}

One case was clinically diagnosed as cardiac sarcoidosis with dilated right ventricular chamber. The initial pathological diagnosis of endomyocardial biopsy in this $>40$ years old man was myocarditis possibly related to systemic lupus erythematosus. He was treated with cyclophosphamide, methylon, cyclosporine $\mathrm{A}$, and azathioprine. MRI findings were compatible with sarcoidosis (Supplementary Fig. S1).

Review of endomyocardial biopsy revealed three foci of a very small collection of 8-10 histiocytes, which was not interpreted as a granuloma at the initial pathologic examination (Fig. 1A).

The resected heart weighed $350 \mathrm{~g}$. There were multiple white scars on the myocardium (Fig. 6). The right ventricular free wall was severely fibrotic and no myocardial tissue was seen at some parts of the right ventricular wall. The trabeculae at the right ventricular apex were severely atrophic and only fibrous cords were noted (Supplementary Fig. S2). An entire plane of the shortaxis of the heart showed large scars scattered at the left and right ventricles and ventricular septum (Fig. 6B). The distribution of the scars was comparable to that of multifocal subendocardial enhancement by cardiac MRI (Supplementary Fig. S1). In the multiple myocardial scars, scattered histiocytes and a few microgranulomas were found (Supplementary Fig. S2).

The second case had hypertrophied right and left ventricles with multifocal fibrosis.

The initial clinical diagnosis of this $>40$ years old man was cardiac amyloidosis. Echocardiography showed a borderline increase in the left ventricular wall thickness with apical regional wall motion abnormalities. Cardiac MRI showed patchy, irregular, and delayed enhancement sparing subendocardium sug- 
gesting non-ischemic cardiomyopathy (Supplementary Fig. S3). PET with 2-deoxy-2- $\left[{ }^{18} \mathrm{~F}\right]$ fluoro- D-glucose (FDG-PET) did not show hot uptake in the myocardium. Holter monitoring showed non-sustained ventricular tachycardia of three beats on two occasions.
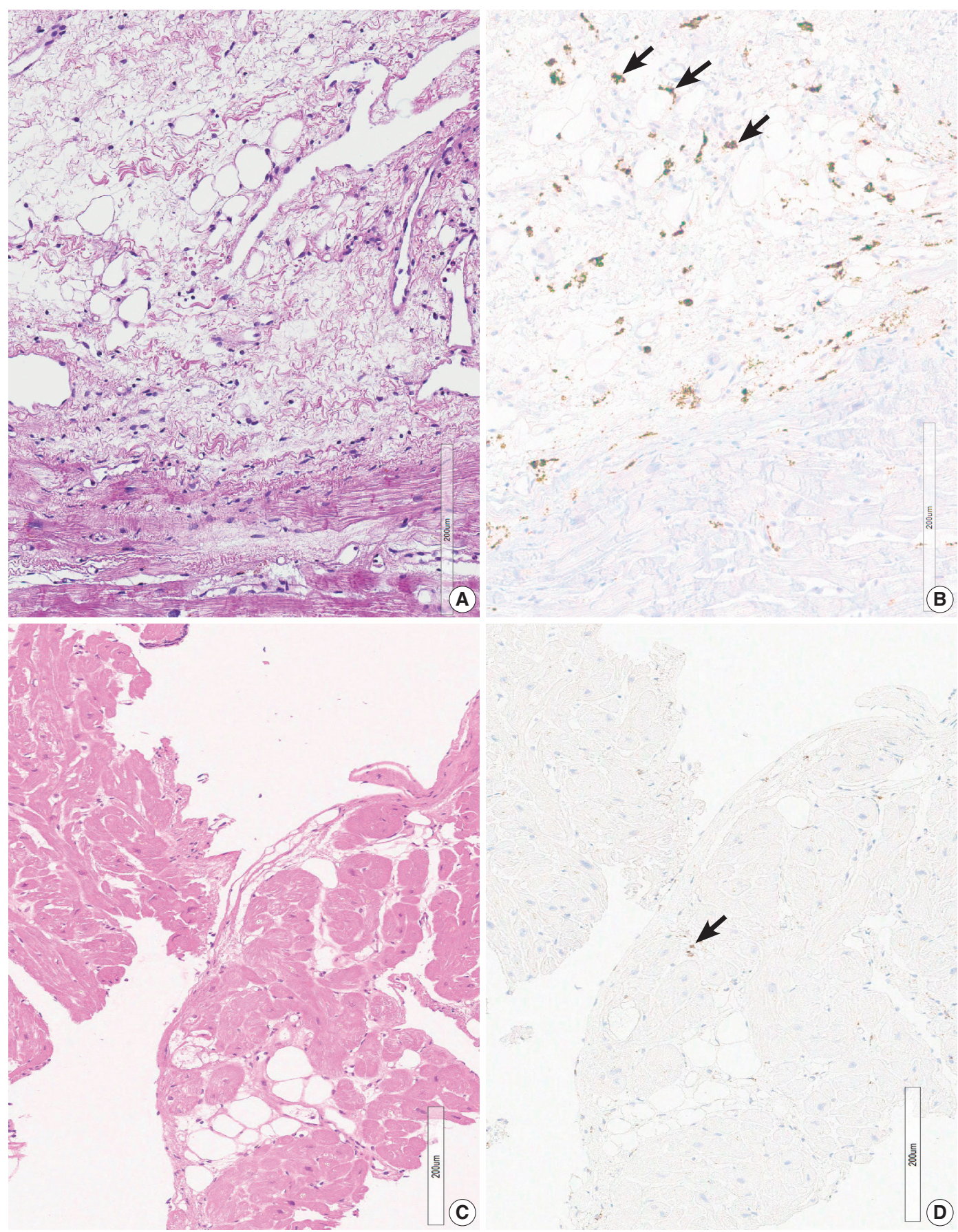

(C)
Endomyocardial biopsy was performed twice at ages 49 and 51. One biopsy at age 51 showed focal patchy fibrosis with a single micro-granuloma (Supplementary Fig. S4).

The resected heart after transplantation at age 51 weighed $560 \mathrm{~g}$. Both right and left ventricular walls were hypertrophied.

B)

Fig. 3. Histiocytic infiltration, confluent fibrosis and fatty change. (A) Confluent fibrosis, associated fatty change within the fibrosis in case 2-1. (B) CD68 staining in the same area shows increased histiocytes (arrows) scattered in the fibrous area. (C) Confluent fibrosis, associated fatty change within the fibrosis in case 3-1. (D) CD68 staining in the same area shows very rare or no increase of histiocytes (arrow) in the fibrous area. 


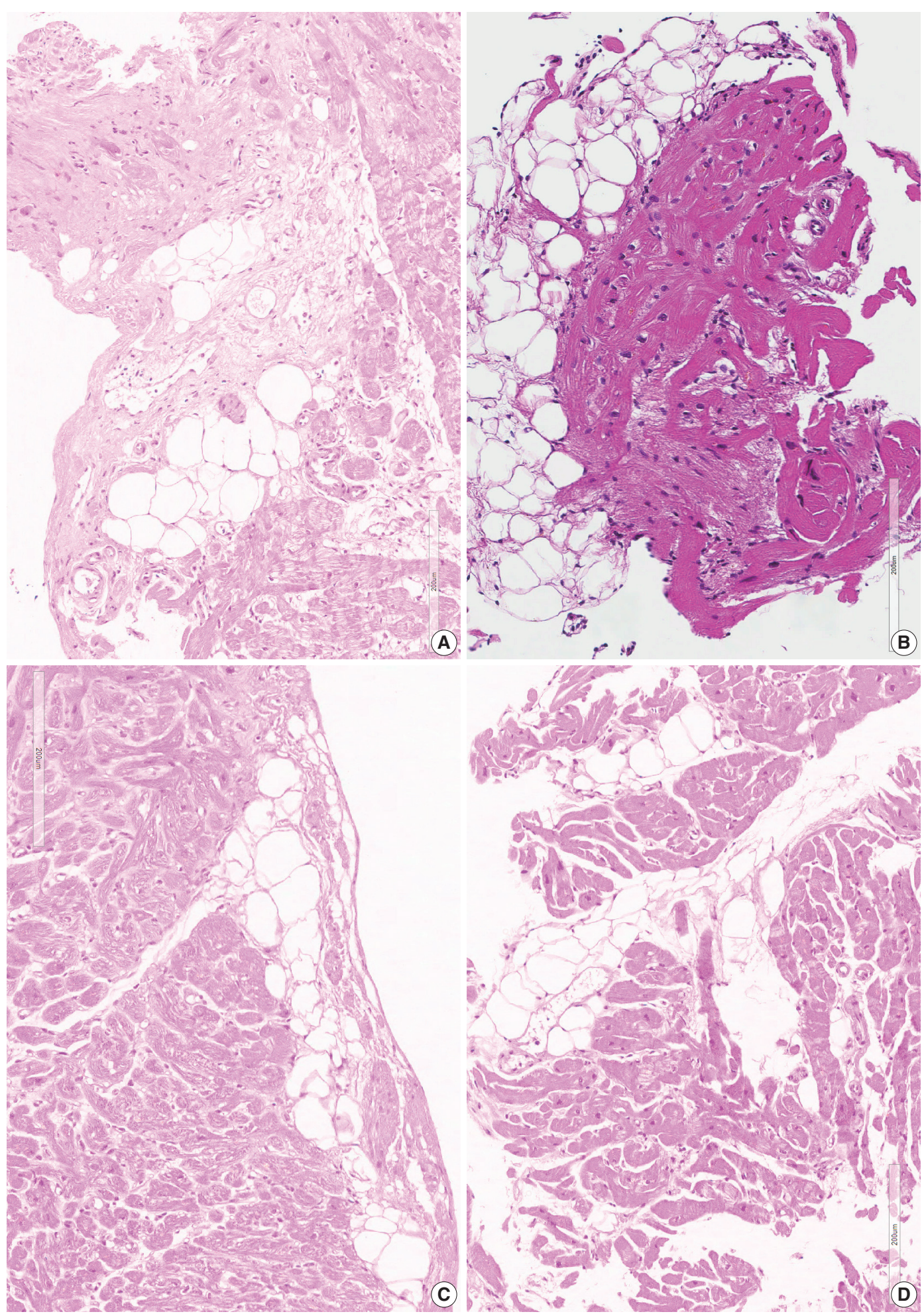

Fig. 4. Different types of fatty changes in endomyocardial biopsies. (A) Fatty infiltration in the background of confluent fibrosis (case 3-2). (B) Fatty tissue with variable sizes of adipocytes and adjacent myocardium also show post-inflammatory fibrosis (case 1-5). (C) Subendocardial deposition of fatty tissue. Slender fibrotic zone is visible at the margin of fatty area (case 3-5). (D) Fatty infiltration between the myocardial bundles. Adjacent myocardium is normal without fibrosis or inflammation (case 5-4). 
There were multiple white scars in the myocardium, particularly in the left ventricle, the ventricular septum, and papillary muscles (Fig. 7). Histologically, the scars were dense fibrous scars and had fatty replacement. At least five micro-granulomas and histiocytes were scattered (Fig. 2).

The third case was clinically suspected as cardiac sarcoidosis
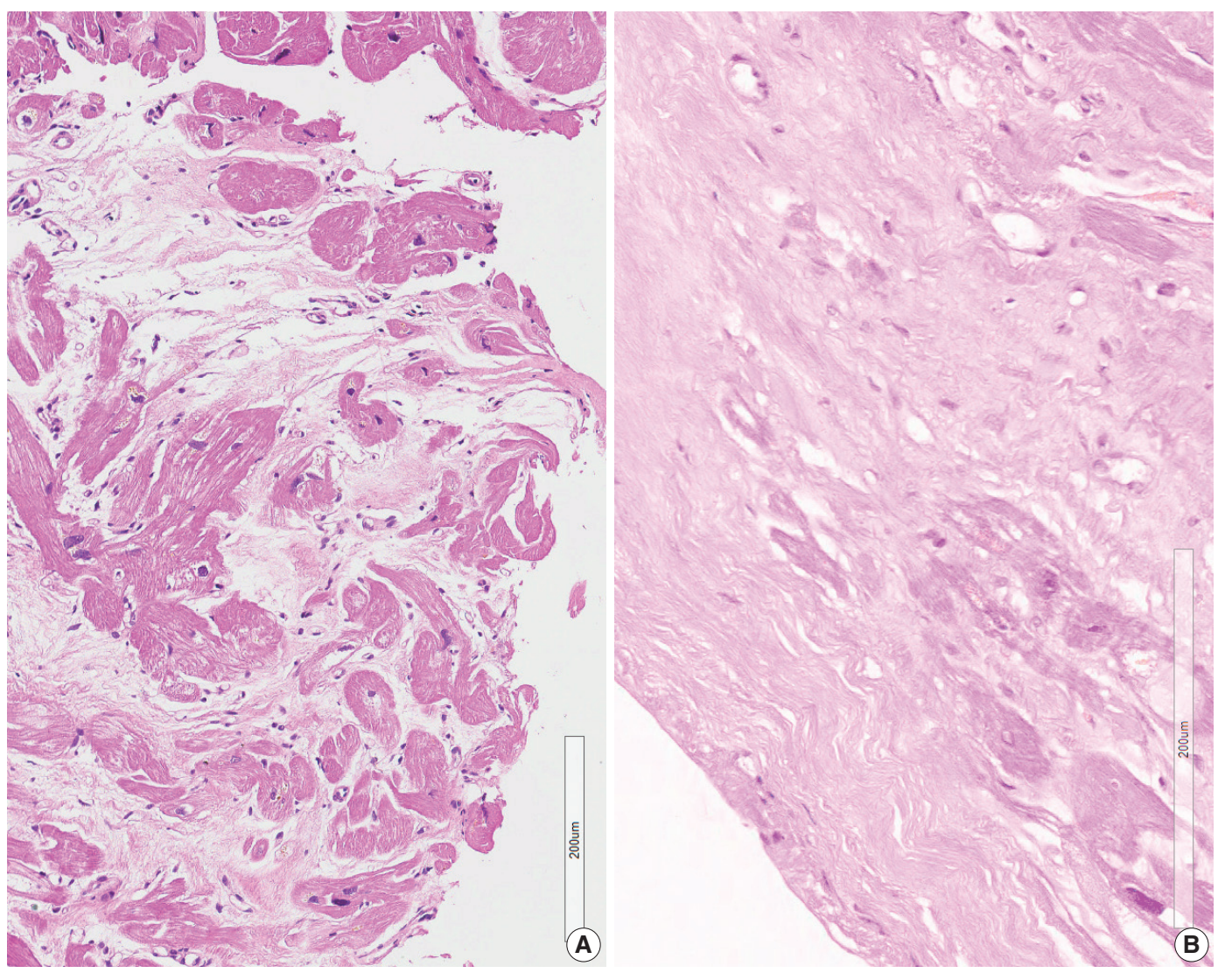

Fig. 5. Confluent fibrosis without associated fatty tissue. (A) Confluent fibrosis is not evident in a small biopsy but fibrosis bigger than five times the diameter of the myocardial cells was interpreted as a scar related to a granuloma (case 4-2). (B) Broad scar at the endocardial zone. Some adipocyte-like spaces were found but the scattered individual spaces were not interpreted as fatty change (Case 4-3).
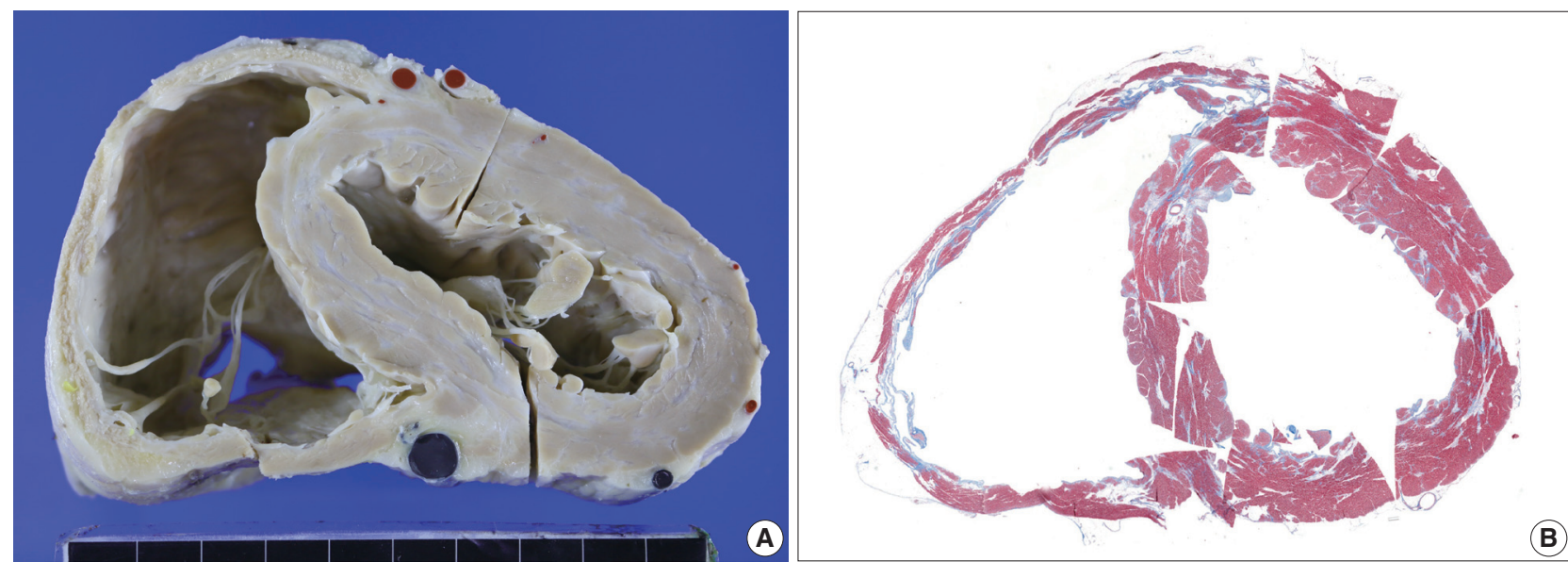

Fig. 6. A short-axis sectional view of the first transplant heart with sarcoidosis and fibrosis. (A) A short-axis sectional view of the heart shows multifocal confluent fibrosis involving both ventricles. The right ventricular thinning and dilatation are prominent. Coronary arteries and cardiac veins are filled with red and blue silicone rubber cast. (B) Histotopographic mapping of a short-axis plane of the heart by Masson's trichrome staining reveals prominent fibrosis (in blue color) in the right ventricular free wall and patchy fibrosis in the ventricular septum and left ventricle. 

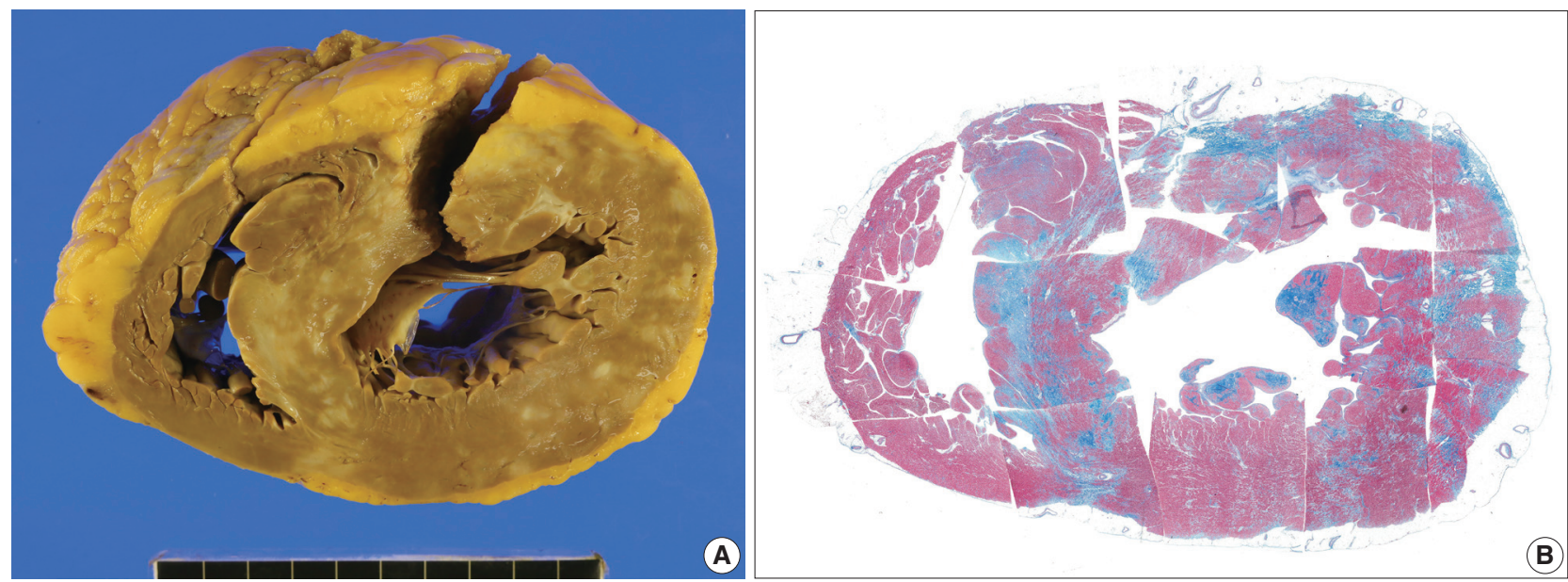

Fig. 7. The second transplant heart with sarcoidosis and hypertrophied ventricles. (A) Sectional view of the heart shows multifocal confluent fibrosis involving predominantly left ventricle and the ventricular septum. Epicardial fatty tissue is prominent in the right ventricle but the myocardium is not much involved. (B) Histotopographic mapping of a short-axis plane of the heart by Masson's trichrome staining reveals prominent fibrosis (in blue color) in the interventricular septum and the left ventricular free wall. Distribution of the fibrosis is prominent but not limited to the subepicardial zone.
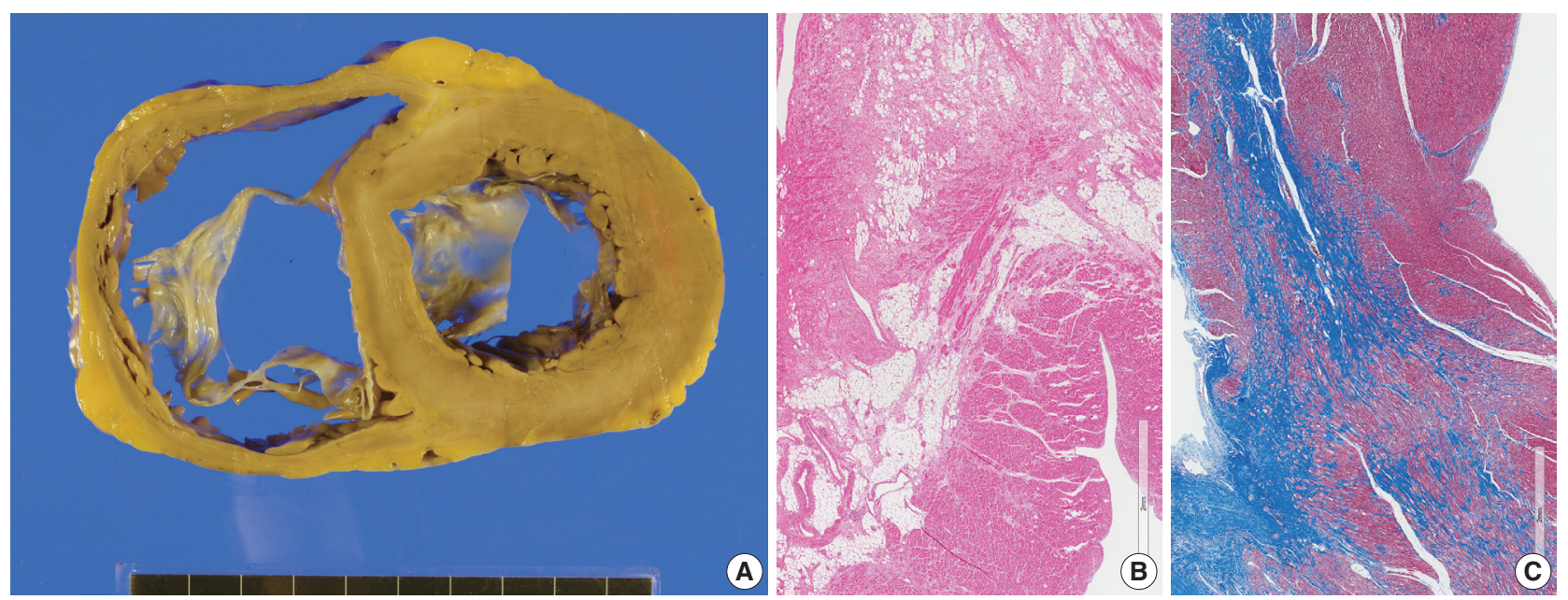

Fig. 8. The third transplant heart mimicking sarcoidosis on microscopy but not likely of sarcoidosis on macroscopic view. (A) The left ventricle is dilated and hypertrophied. Multifocal and diffuse fibrosis was noted on gross examination. Fatty infiltration was prominent at the anterior part of the ventricular septum and mid-septal fibrosis was evident. (B) Fatty change associated with myocardial fibrosis was seen at the anterior part of the ventricular septum. (C) Low magnification of the mid-septal fibrosis (Masson's trichrome stain).

but pathological study denied the diagnosis after examination of the resected heart. The initial clinical presentation of a $>40$ years old man was dyspnea on exertion and edema in the lower extremities. Complete atrioventricular block was noted, and a permanent pacemaker was inserted. Heart transplantation was performed at age 45 . MRI was not performed, but ${ }^{18}$ F-FDG-PET revealed a localized hypermetabolic lesion at the apex and apical and midinferior anteroseptal wall. Computed tomography angiography showed no significant myocardial fibrosis.

The endomyocardial biopsy revealed three large pieces with different features. One piece showed confluent fibrosis and focal fatty infiltration. The second and third pieces were relatively well-preserved myocardium with interstitial fibrosis.

The resected heart weighed $365 \mathrm{~g}$ and both ventricles were enlarged. There was multifocal fibrosis in the left ventricular wall and septum. The mid-septal fibrosis was prominent and was diffuse rather than patchy. Fatty infiltration was seen at the anterior end of the basal part of the ventricular septum (Fig. 8). The fibrosis pattern was diffuse in the myocardium, but dense hyalinized fibrosis was seen in the mid-septal area (Fig. 8). We interpreted this explant heart as non-specified dilated cardiomyopathy rather than sarcoidosis. 
An autopsy case (a man who died suddenly and a heart with many classical granulomas)

We reviewed a previously reported autopsied heart with sarcoidosis [19]. Macroscopic findings of the classical cardiac sarcoid lesion and histologic details are enclosed as supplementary materials. This 43-year-old man presented with syncope without any significant clinical history. Forensic autopsy revealed enlarged lymph nodes with sarcoid granuloma. The heart weighed $490 \mathrm{~g}$ and there were several conglomerated mass-like lesions $(5.0 \times$ $1.2 \mathrm{~cm}$ ) at the anterior wall of the left ventricle adjacent to the left anterior descending coronary artery (Supplementary Fig. S5). Histological examination showed active non-caseating granulomata involving multiple sites in the left and right ventricles. Lesions were subepicardial, subendocardial, or transmural in the ventricular wall (Supplementary Fig. S6). Fatty infiltration or fatty replacement was also noted even in the deep myocardium. Immunohistochemistry revealed CD68-positive histiocytes and CD3-positive T-lymphocytes (Supplementary Fig. S7).

\section{DISCUSSION}

Presence of classical granuloma in cardiac tissue is considered as the gold standard for diagnosis of sarcoidosis although infectious and immunologic causes are suggested in some cases [20,21]. Pathologists are asked to report the presence or absence of granulomas in small endocardial biopsies, and granulomas are not seen in most biopsies. When we don't see any granulomas, it would be more useful for clinicians whether the case has some indirect or suggestive finding, rather than excluding the case from pathological diagnosis of sarcoidosis. We believe four histological findings and their combinations will have some value for pathological support of clinical practice on patients with cardiac sarcoidosis.
We compared our findings with radiological findings (Table 2). The presence of micro-granulomas (four cases, excluding a case without MRI findings) or increased histiocytic infiltration (two biopsies from one patient) was always (100\%) associated with fatty infiltration and confluent fibrosis, and these cases showed radiological features of probable sarcoidosis. Among six cases with fatty change and confluent fibrosis, five cases had MRI findings. The MRI findings of three cases indicated probable cardiac sarcoidosis (3/5 or $60 \%$ possibility of sarcoidosis by radiology). When a single finding of either confluent fibrosis or fatty change was present, three of nine cases (33\%) radiologically supported sarcoidosis. From these observations, we can categorize patients with two (fatty change and confluent fibrosis) or more indicators as cases with probable sarcoidosis on endomyocardial biopsy.

Clinical findings were so diverse that they matched poorly with pathological or radiological findings. None of the six cases with confirmed granulomas or increased histiocytes showed heart block. Five among 12 cases ( $42 \%$ ) with pathological features of more than two indicators (confluent fibrosis and fatty change) had heart block. There were seven cases with heart block and five of them $(71 \%)$ were probable for cardiac sarcoidosis by our pathological criteria.

In regard to corticosteroid treatment on cardiac sarcoidosis, cases at early inflammatory phase will get more benefit than cases with end-stage fibrotic lesions $[22,23]$. The detection of active definitive granulomas would be a definitive indication for steroid treatment. It is not surprising therefore that there was a poor correlation with the pathological parameters in this series.

The non-caseating granuloma is the classic histopathology of sarcoidosis $[1,24]$ but there exists a spectrum of histology. Upon review of autopsy cases of cardiac sarcoidosis, granulomatous lesions were found in most of cases (108 patients) but in some cases (5 patients) myocardial scarring was a dominant lesion [18].

Table 2. Numbers of cases in our interpretation categories and radiologic features

\begin{tabular}{|c|c|c|c|c|c|c|}
\hline \multirow{2}{*}{ Our interpretation categories ${ }^{a}$} & \multicolumn{6}{|c|}{ MRI finding } \\
\hline & Probable & Possible & Nonspecific & Unlikely & Not checked & Total \\
\hline 1. Positive for cardiac sarcoidosis: presence of four indicators & 4 & - & - & - & 1 & 5 \\
\hline $\begin{array}{l}\text { 2. Probable for cardiac sarcoidosis (1): Presence of three (confluent fibrosis, } \\
\text { fatty change and increased histiocytes) but no micro-granuloma }\end{array}$ & 2 & - & - & - & - & 2 \\
\hline $\begin{array}{l}\text { 3. Probable for cardiac sarcoidosis (2): Presence of confluent fibrosis and fatty } \\
\text { tissue infiltration }\end{array}$ & 3 & 1 & 1 & - & 1 & 6 \\
\hline 5. Nonspecific (2): Fatty tissue without associated fibrosis & 1 & - & 3 & 2 & - & 6 \\
\hline 6. Nonspecific (3): None of four possible indicators on endomyocardial biopsy & - & - & 3 & 1 & 1 & 5 \\
\hline Total & 12 & 1 & 8 & 3 & 3 & 27 \\
\hline
\end{tabular}

Four presumptive indicators of cardiac sarcoidosis are micro-granuloma, confluent fibrosis, fatty change and increased histiocytes.

$\mathrm{MRI}$, magnetic resonance imaging.

aDetails of case groups 1-6 are shown in Table 1. 
Histologic features were variable and the spectrum was divided into four types: exudative type, granuloma type, combined type, and fibrotic type (Table 3) [25]. Some extreme examples presented as cases without granuloma in any internal organs at autopsy, which had several years history of cardiac arrhythmia, and where the initial histological diagnosis was sarcoidosis in the lymph node [18]. Our cases with very small granuloma-like lesions on endomyocardial biopsy were interpreted as myocarditis or ignored instead of being interpreted as granulomas. Each granuloma was composed of 5-10 cells, and giant cells were not found.

A micro-granuloma is a type of small granuloma or granuloma-like lesion. The micro-granuloma was described in a medical dictionary as "a term of art referring to an aggregate of less than 25 epithelioid histiocytes" [26] and is associated with Crohn's disease [27]. The micro-granuloma in our new definition was smaller than that of the dictionary. We further suggested the increase in scattered histiocytes as a variant of a lesion with similar significance of granuloma in cardiac sarcoidosis. A recent study revealed that the presence of CD3-, CD68-, and CD163-positive cells in endomyocardial biopsies of patients with dilated cardiomyopathy was associated with cardiac fibrosis and poor clinical outcomes [28]. The increase in CD68-positive cells may be an indicator of active myocardial inflammatory conditions, including sarcoidosis.

It is well documented that the histologic features of sarcoid granuloma are resolved by corticosteroid treatment $[29,30]$ and spontaneous regression was observed in one-third of pulmonary sarcoidosis [31]. The case of cardiac sarcoidosis without granuloma at autopsy was interpreted as a result of steroid treatment in a previous report [18]. As is shown in the variable features of granulomas in the cardiac sarcoidosis on autopsied heart (Supplementary Fig. S7), presence of giant cells and histiocytes varies in the lesions and they will resolve to fibrotic lesions. Therefore, we would interpret the stromal micro-granuloma-like lesions as a healed stage of collection of scattered histiocytes.

The significance of confluent scars in the myocardium is also debatable. We agree that they are large replacement fibrosis [32] but they are different from the large scars of myocardial infarct by their distribution related to the coronary arterial supply. It is not always possible to obtain a large specimen on endomyocardial biopsy to find confluent fibrosis but it was possible in some cases. Macroscopic classification of myocardial lesions was described in three classes: spotty pattern, conglomerate band-like pattern, and dendritic pattern (Table 3) [25]. The detection of such patchy fibrosis may indicate the pathologic substrates of an apparent conduction delay of the electrical impulse and fractionation due to asynchronous activation in different tracts [32]. We therefore interpret large scars as probable evidence for cardiac sarcoidosis.

The presence of mature fat or "fibro-fatty replacement of myocardium" in endomyocardial biopsies is a hallmark of arrhythmogenic right ventricular cardiomyopathy [33-35]. However, the presence of mature epicardial fat in the endomyocardial biopsy is common. In such samples, we observed completely normal fatty tissue with direct attachment to the normal myocardium (Fig. 4D). Careful observation of fatty tissue in our endomyocardial biopsies revealed some different microscopic features, including fatty tissue associated with fibrosis and histiocytes. These fat cells often had small or variable cytoplasmic contours. We interpreted these as recently transformed fatty changes at the endocardial zone, or deep from the pericardium. It is not easy to differentiate these post-inflammatory fatty changes from those seen in the old myocardial infarcts. Fatty changes alone are not significant, but if fatty change is associated with young scars and histiocytes, it may be a clue to sarcoidosis.

Table 3. Macroscopic and histological classification on the spectrum of pathology in the cardiac sarcoidosis and cardiac fibrosis in the literature

\begin{tabular}{l} 
Morphologic patterns \\
\hline 1. Macroscopic classification of myocardial lesions in cardiac sarcoidosis [25] \\
- Spotty pattern \\
- Conglomerate band-like pattern \\
- Dendritic pattern \\
2. Histologic features of myocardial lesions in cardiac sarcoidosis [25] \\
- Exudative type: marked lymphocytic infiltration, diffuse edema, collection of histiocytes in the interstitium \\
- Granuloma type: typical epithelioid-cell-granuloma formation with giant cells and lymphocytes \\
- Combined type: some atrophic epithelioid-cell-granulomatous and fibrous change \\
- Fibrotic type: the myocardial tissue replaced by fibro-hyaline changes, with sparse lymphocytic infiltration. \\
3. Phases of the lesion in cardiac sarcoidosis [36] \\
- Early (primarily lymphocytic) phase: areas indistinguishable from lymphocytic myocarditis \\
- Intermediate (primarily granulomatous) phase: active granulomatous lesion \\
- Late (primarily scar) phase: areas composed predominantly of scar
\end{tabular}


When we diagnose the resected heart for cardiac transplantation or autopsy, we should evaluate the patient as a whole. We should include any previous pathologic processes in our final diagnosis. We would interpret hearts without definitive granuloma as a case of cardiac sarcoidosis if there is presence of microgranulomas and macroscopic distribution of the lesion. The macroscopic distribution of cardiac lesions in our cases matches very well with the radiologic findings. There were scattered macroscopic fibrotic scars in the ventricular septum, free wall, sub-epicardium, and papillary muscles without any matching distribution in the coronary arterial supply $[10,18,26]$. This random pattern was also present in the autopsied heart with classical histologic features of sarcoidosis. If radiologic diagnosis of sarcoidosis is based on the macroscopic distribution of the lesion, the same feature of the resected heart can form the pathologic basis of cardiac sarcoidosis when we diagnose cardiac sarcoidosis for whole heart specimen. The macroscopic distribution of the granulomas was described as patchy and involving the ventricular septum and the subepicardium [36,37]. These features are confirmed by radiologic studies [38-40].

Differential diagnosis of cardiac sarcoidosis from the pathologists' view was reviewed $[1,24]$. When we observe granulomas, we suspect sarcoidosis, giant cell myocarditis, and other granulomatous myocardial lesions, including those of infectious origin $[20,21]$. It may be necessary to broadly categorize these cases so that they are all classified into "granulomatous myocarditis." if any specific causes are detected. In our experience with cases involving clinically suspected cardiac sarcoidosis, the pathologists are asked if the case is compatible with cardiac sarcoidosis, and to carefully examine the pathologic specimens to find any suggestive features and any small micro-granulomas. This attitude will support cardiologists in considering cardiac sarcoidosis to ensure they do not miss the chance to start optimal treatment. There are several different patterns of myocardial inflammatory lesions $[1,41]$. In general, viral myocarditis involves the heart as a diffuse lesion. The lesion may involve some parts more severely, but the margin of the inflammatory lesion is indistinct. Rheumatic myocarditis also has distinct features of involvement of the valve, pericardium, and perivascular interstitium. Infectious myocarditis involves only the focus of involvement. Tuberculous lesions involve predominantly pericardium first, and the myocardial lesions are an extension of pericarditis. For sarcoidosis, nodular and mass-like involvement is characteristic and the intervening myocardium between granulomata is generally spared. It has to be emphasized however that the end-stage fibrotic lesion of cardiac sarcoidosis will show different shape. We value the endo- myocardial biopsy to exclude other differential diagnoses from sarcoidosis in the absence of granulomatous inflammation.

Explant hearts for transplantation have similar concerns. When cardiac sarcoidosis is clinically diagnosed, pathologists will check for micro-granuloma. It is worthwhile to remind pathologists that granulomas may be indistinct in cardiac sarcoidosis after treatment. High suspicion for cardiac sarcoidosis is necessary in patients with end-stage heart failure with pacemaker or implantable cardiac defibrillator [42]. Cardiologists and surgeons may incorrectly diagnose dilated cardiomyopathy or ischemic cardiomyopathy when the case was not associated with rhythm disturbance. However, involvement of the conduction system is not the rule in cardiac sarcoidosis $[1,18,24,25]$. The pathologists' suspicion for cardiac sarcoidosis will require a search to find a granuloma or a micro-granuloma to confirm the diagnosis. It is also important for pathologists to examine the macroscopic morphology of the explant heart to find large confluent scars and fatty changes in the myocardium in both subendocardium and subepicardium.

\section{Supplementary Information}

The Data Supplement is available with this article at https://doi.org/10.4132/ jptm.2020.06.10.

\section{Ethics Statement}

The study plan of this research was reviewed by the Seoul National University College of Medicine/Seoul National University Hospital (IRB NO. H-1802-050-921 and RESEARCH TITLE: Indirect pathological indicators for cardiac sarcoidosis on endomyocardial biopsy or the explant heart for transplantation). Review comments was: Since the risk of research is minimal, it is for expedited review, and the statement of reason for waiver of informed consent is reasonable. According to IRB Approval Criteria, the IRB approves the research. Further details of the rationale for this waiver was that this research was based on retrospective review of medical record and pathology slides, and anonymized case analysis. This analysis did not alter management of patients. See Attachment D: Informed consent and waiver of consent (https:// www.hhs.gov/ohrp/sachrp-committee/recommendations/2013-january10-letter-attachment-d/index.html).

\section{ORCID}

Myung-Jin Cha https://orcid.org/0000-0001-6180-0157 Jeong-Wook Seo https://orcid.org/0000-0003-0242-1805 Seil Oh https://orcid.org/0000-0002-2504-9615 Eun-Ah Park https://orcid.org/0000-0001-6203-1070 Sang-Han Lee https://orcid.org/0000-0003-0390-3494 Moon Young Kim https://orcid.org/0000-0002-2501-2206 Jae-Young Park https://orcid.org/0000-0003-3893-0521

\section{Author Contributions}

Conceptualization: MJC, JWS, SO. Data curation: MJC, JWS, SO, EAP, SHL, MYK, JYP. Formal analysis: MJC, JWS, SO, EAP, SHL. Funding acquisition: JWS, SO, SHL. Investigation: MJC, JWS, SO, EAP, SHL, MYK, JYP. Methodology: MJC, JWS, SO, EAP, SHL, MYK, JYP. Project administration: JWS, SO. Resources: MJC, JWS, SO, EAP, SHL, MYK, JYP. Super- 
vision: JWS, SO. Visualization: MJC, JWS. Writing_original draft: MJC, JWS. Writing—review \& editing: MJC, JWS, SO, EAP, SHL.

\section{Conflicts of Interest}

The authors declare that they have no potential conflicts of interest.

\section{Funding Statement}

No funding to declare.

\section{Acknowledgments}

All authors wish to thank Kyeong Hee Seo on her editing services of photographs.

\section{References}

1. Lagana SM, Parwani AV, Nichols LC. Cardiac sarcoidosis: a pathology-focused review. Arch Pathol Lab Med 2010; 134: 1039-46.

2. Hamzeh N, Steckman DA, Sauer WH, Judson MA. Pathophysiology and clinical management of cardiac sarcoidosis. Nat Rev Cardiol 2015; 12: 278-88.

3. Hulten E, Aslam S, Osborne M, Abbasi S, Bittencourt MS, Blankstein R. Cardiac sarcoidosis-state of the art review. Cardiovasc Diagn Ther 2016; 6: 50-63.

4. Newman LS, Rose CS, Maier LA. Sarcoidosis. N Engl J Med 1997; 336: 1224-34.

5. Kang EH. Sarcoidosis in Korea: revisited. J Korean Med Assoc 2008; 51: 925-32.

6. Mehta D, Lubitz SA, Frankel Z, et al. Cardiac involvement in patients with sarcoidosis: diagnostic and prognostic value of outpatient testing. Chest 2008; 133: 1426-35.

7. Longcope WT, Freiman DG. A study of sarcoidosis; based on a combined investigation of 160 cases including 30 autopsies from The Johns Hopkins Hospital and Massachusetts General Hospital. Medicine (Baltimore) 1952; 31: 1-132.

8. Silverman KJ, Hutchins GM, Bulkley BH. Cardiac sarcoid: a clinicopathologic study of 84 unselected patients with systemic sarcoidosis. Circulation 1978; 58: 1204-11.

9. Iwai K, Takemura T, Kitaichi M, Kawabata Y, Matsui Y. Pathological studies on sarcoidosis autopsy. II. Early change, mode of progression and death pattern. Acta Pathol Jpn 1993; 43: 377-85.

10. Sayah DM, Bradfield JS, Moriarty JM, Belperio JA, Lynch JP 3rd. Cardiac involvement in sarcoidosis: evolving concepts in diagnosis and treatment. Semin Respir Crit Care Med 2017; 38: 477-98.

11. Chapelon-Abric C, de Zuttere D, Duhaut P, et al. Cardiac sarcoidosis: a retrospective study of 41 cases. Medicine (Baltimore) 2004; 83: 315-34.

12. Birnie DH, Sauer WH, Bogun F, et al. HRS expert consensus statement on the diagnosis and management of arrhythmias associated with cardiac sarcoidosis. Heart Rhythm 2014; 11: 1305-23.

13. Cooper LT, Baughman KL, Feldman AM, et al. The role of endomyocardial biopsy in the management of cardiovascular disease: a scientific statement from the American Heart Association, the American College of Cardiology, and the European Society of Cardiology Endorsed by the Heart Failure Society of America and the Heart Failure Association of the European Society of Cardiology. Eur Heart J 2007; 28: 3076-93.

14. Hiraga H, Yuwai K, Hiroe M. Diagnostic standard and guidelines for sarcoidosis. Jpn J Sarcoidosis Granulomatous Disord 2007; 27: 89-102.
15. Bhimaraj A, Trachtenberg B, Valderrabano M. Robotically guided left ventricular biopsy to diagnose cardiac sarcoidosis: a multidisciplinary innovation leading to first-in-human case. Circ Heart Fail 2018; 11: e004627.

16. Liang JJ, Hebl VB, DeSimone CV, et al. Electrogram guidance: a method to increase the precision and diagnostic yield of endomyocardial biopsy for suspected cardiac sarcoidosis and myocarditis. JACC Heart Fail 2014; 2: 466-73.

17. Casella M, Dello Russo A, Vettor G, et al. Electroanatomical mapping systems and intracardiac echo integration for guided endomyocardial biopsy. Expert Rev Med Devices 2017; 14: 609-19.

18. Roberts WC, McAllister HA Jr, Ferrans VJ. Sarcoidosis of the heart. A clinicopathologic study of 35 necropsy patients (group 1) and review of 78 previously described necropsy patients (group 11). Am J Med 1977; 63: 86-108.

19. Lee SK, Kim SZ, Kum YS, et al. Sudden death from cardiac sarcoidosis: a case report. Korean J Pathol 2003; 37: 358-61.

20. Asakawa N, Uchida K, Sakakibara M, et al. Immunohistochemical identification of Propionibacterium acnes in granuloma and inflammatory cells of myocardial tissues obtained from cardiac sarcoidosis patients. PLoS One 2017; 12: e0179980.

21. Fang C, Huang H, Xu Z. Immunological evidence for the role of mycobacteria in sarcoidosis: a meta-analysis. PLoS One 2016; 11: e0154716.

22. Ratner SJ, Fenoglio JJ Jr, Ursell PC. Utility of endomyocardial biopsy in the diagnosis of cardiac sarcoidosis. Chest 1986; 90: 528-33.

23. Yodogawa K, Seino Y, Ohara T, Takayama H, Katoh T, Mizuno K. Effect of corticosteroid therapy on ventricular arrhythmias in patients with cardiac sarcoidosis. Ann Noninvasive Electrocardiol 2011; 16: 140-7.

24. Bagwan IN, Hooper LV, Sheppard MN. Cardiac sarcoidosis and sudden death: the heart may look normal or mimic other cardiomyopathies. Virchows Arch 2011; 458: 671-8.

25. Matsui Y, Iwai K, Tachibana T, et al. Clinicopathological study of fatal myocardial sarcoidosis. Ann N Y Acad Sci 1976; 278: 455-69.

26. Segen JC. The dictionary of modern medicine. Huntington Valley: Farlex Inc., 2011.

27. Rotterdam H, Korelitz BI, Sommers SC. Microgranulomas in grossly normal rectal mucosa in Crohn's disease. Am J Clin Pathol 1977; 67: 550-4.

28. Nakayama T, Sugano Y, Yokokawa T, et al. Clinical impact of the presence of macrophages in endomyocardial biopsies of patients with dilated cardiomyopathy. Eur J Heart Fail 2017; 19: 490-8.

29. Sullivan RD, Mayock RL, Jones R Jr. Local injection of hydrocortisone and cortisone into skin lesions of sarcoidosis. J Am Med Assoc 1953; 152: 308-12.

30. Sharma OP. Pulmonary sarcoidosis and corticosteroids. Am Rev Respir Dis 1993; 147(6 Pt 1): 1598-600.

31. Kim MS, Park CK, Shin HJ, et al. Review of sarcoidosis in a province of South Korea from 1996 to 2014. Tuberc Respir Dis 2017; 80: 291-5.

32. de Jong S, van Veen TA, van Rijen HV, de Bakker JM. Fibrosis and cardiac arrhythmias. J Cardiovasc Pharmacol 2011; 57: 630-8.

33. Terasaki F, Ishizaka N. Cardiac sarcoidosis and arrhythmogenic right ventricular cardiomyopathy: potential differential diagnoses for arrhythmogenic ventricular cardiomyopathy. Intern Med 2016; 55: 1041-2.

34. Marcus FI, McKenna WJ, Sherrill D, et al. Diagnosis of arrhythmo- 
genic right ventricular cardiomyopathy/dysplasia: proposed modification of the task force criteria. Circulation 2010; 121: 1533-41.

35. Ponsiglione A, Puglia M, Morisco C, et al. A unique association of arrhythmogenic right ventricular dysplasia and acute myocarditis, as assessed by cardiac MRI: a case report. BMC Cardiovasc Disord 2016; 16: 230.

36. Tavora F, Cresswell N, Li L, Ripple M, Solomon C, Burke A. Comparison of necropsy findings in patients with sarcoidosis dying suddenly from cardiac sarcoidosis versus dying suddenly from other causes. Am J Cardiol 2009; 104: 571-7.

37. Shirani J, Roberts WC. Subepicardial myocardial lesions. Am Heart J 1993; 125(5 Pt 1): 1346-52.

38. Yang Y, Safka K, Graham JJ, et al. Correlation of late gadolinium enhancement MRI and quantitative T2 measurement in cardiac sarcoidosis. J Magn Reson Imaging 2014; 39: 609-16.

39. Shimada T, Shimada K, Sakane T, et al. Diagnosis of cardiac sarcoidosis and evaluation of the effects of steroid therapy by gadolinium-DTPA-enhanced magnetic resonance imaging. Am J Med 2001; 110: 520-7.

40. Smedema JP, Snoep G, van Kroonenburgh MP, et al. Evaluation of the accuracy of gadolinium-enhanced cardiovascular magnetic resonance in the diagnosis of cardiac sarcoidosis. J Am Coll Cardiol 2005; 45: 1683-90.

41. Rosen Y. Pathology of sarcoidosis. Semin Respir Crit Care Med 2007; 28: 36-52.

42. Sun BJ, Lee PH, Choi HO, et al. Prevalence of echocardiographic features suggesting cardiac sarcoidosis in patients with pacemaker or implantable cardiac defibrillator. Korean Circ J 2011; 41: 313-20. 